\title{
Linx
}

Revue des linguistes de l'université Paris X Nanterre

46 | 2002

Les connecteurs

\section{Les conjonctions causales et la propriété d'enchâssement}

Sophie Hamon

\section{(2) OpenEdition}

\section{Journals}

Édition électronique

URL : http://journals.openedition.org/linx/88

DOI : $10.4000 / \operatorname{lin} x .88$

ISSN : 2118-9692

\section{Éditeur}

Presses universitaires de Paris Nanterre

\section{Édition imprimée}

Date de publication : 1 juin 2002

Pagination : 25-35

ISSN : 0246-8743

\section{Référence électronique}

Sophie Hamon, "Les conjonctions causales et la propriété d'enchâssement », Linx [En ligne],

46 | 2002, mis en ligne le 24 janvier 2011, consulté le 02 mai 2019. URL : http://

journals.openedition.org/linx/88; DOI : 10.4000/linx.88

Département de Sciences du langage, Université Paris Ouest 


\title{
Les conjonctions causales et la propriété d'enchâssementı
}

\author{
Sophie Hamon, Université de Paris X-Nanterre
}

\section{1- La propriété d'enchâssement d'une phrase complexe}

Dans le cadre d'une étude des propriétés formelles qui distinguent les conjonctions dites de cause $^{2}$ (principalement car, parce que, puisque, vu que et étant donné que), la question de l'enchâssement est incontournable. Cette propriété de subordination permet d'ailleurs de séparer, dans les énoncés, ceux que l'on reconnaîtra comme "phrases »; ainsi, Au secours! ("Elle a crié qu'au secours.) n'est pas une phrase. Appliquée aux conjonctions causales, elle se définit comme suit : si un énoncé, contenant une proposition A et une proposition causale B, est enchâssé dans une autre proposition C, alors deux cas de figure sont possibles :

i) ou bien la proposition causale n'est pas comprise dans l'enchâssement et donc s'interprète comme portant sur l'ensemble formé par C + A (ex.1);

ii) ou bien la proposition causale est comprise dans l'enchâssement et donc s'interprète comme ne portant que sur A (ex.2).

1- [Julie sait que Paul a mangé tout le gâteau ] [parce qu'elle l'a vu]

(C

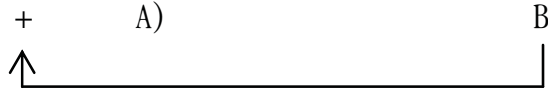

2- Julie sait que [Paul a mangé tout le gâteau parce quil avait faim].

$\mathrm{C}$

(A

$+$

B)

$\uparrow$

Plusieurs tests mettent en évidence la différence syntaxique et interprétative entre (1) et (2). Premièrement, si la proposition $B$ est enchâssée, alors la proposition enchâssante $C$ peut être

\footnotetext{
${ }^{1}$ Je tiens à remercier Danielle Leeman pour ses suggestions et remarques.

${ }^{2}$ En ce qui concerne la traditionnelle distinction entre les conjonctions de coordination et les conjonctions de subordination, cf. M. Grevisse (1986), M. Riegel et al. (1994).
} 
supprimée sans que l'interprétation de la causale soit bouleversée, du fait que la causale B n'est liée logiquement qu'à A. Tel est le cas en (2) et non en (1), où l'énoncé reste curieux ${ }^{3}$ :

1a- * ? Paul a mangé tout le gâteau parce qu'elle l'a vu.

2a- Paul a mangé tout le gâteau parce qu'il avait faim.

Deuxièmement, si B est enchâssé, alors A et B forment un bloc syntaxique et interprétatif tel qu'il ne peut être désolidarisé : la proposition A est difficilement pronominalisable (ex. 2b). Quand il y a détachement, A peut, en revanche, être pronominalisé dans l'enchâssante $\mathrm{C}$, sans que la relation causale soit perturbée (ex. 1b) :

1b- Julie le sait parce qu'elle l'a vu.

2b- * ? Julie le sait parce qu'il avait faim.

L. Gosselin (1993) a utilisé d'autres critères, pour mettre en évidence le détachement ou l'insertion d'une causale par rapport à la portée de la négation, qui peuvent également s'appliquer à la propriété qui nous préoccupe ici. Une proposition est dite détachée :

- s'il y a antéposition de la circonstancielle :

1c- Parce qu'elle l'a vu, Julie sait que Paul a mangé tout le gâteau.

1d- *Julie sait que, parce qu'elle l'a vu, Paul a mangé tout le gâteau. ${ }^{4}$

2c- ?* Parce qu'il avait faim, Julie sait que Paul a mangé tout le gâteau.

2d- Julie sait que, parce qu'il avait faim, Paul a mangé tout le gâteau.

On voit ainsi que la causale ne peut être antéposée que conjointement à la proposition à laquelle elle s'applique.

- si la causale accepte l'insertion d'une incise parenthétique ou d'un adverbe d'évaluation subjective :

1e- Julie sait que Paul a mangé tout le gâteau parce que, justement / paraît-il, elle l'a vu.

2e- ??Julie sait que Paul a mangé tout le gâteau parce que, justement / paraît-il, il avait faim. ${ }^{5}$

D'après les analyses linguistiques du Groupe $\lambda-1$ (1975), il est établi que parce que est la seule conjonction susceptible de présenter les deux cas (i) et (ii). Les conjonctions car et prisque (mais aussi vu que et étant donné que) ne peuvent pas, quant à elles, illustrer la propriété (ii) ${ }^{6}$. Ces analyses utilisent les exemples suivants pour illustrer leurs propos :

3a- Je suis sûr qu'il est là, puisque sa voiture est en bas.

3b - Je compris qu'il était parti, car sa voiture n'était pas là.

Les énoncés (3a) et (3b) sont considérés comme des « phrases dans lesquelles on a seulement enchâssé p et non ( $\mathrm{p}$ car q) ni (p puisque q) : les membres de phrases introduits par car et puisque sont sentis comme l'explication ou la justification de Jai compris que p et Je suis sûr que p et non plus de il était parti ou de il est là. » (Groupe $\lambda-1,1975$, p.252).

\footnotetext{
${ }^{3}$ L'énoncé (1a) est seulement interprétable dans le contexte d'un discours métalinguistique de type : Ecoute, si je te dis que $\mathrm{A}$, c'est parce que $\mathrm{B}$ (construction qui se révèle d'ailleurs curieuse appliquée à (2b)). Ou bien, (1a) change complètement de sens par rapport à (1) : la raison pour laquelle Paul a mangé tout le gâteau est que Marie l'a vu.

${ }^{4}$ Dans ce cas, l'interprétation n'est pas la même, cf. note 1 .

${ }^{5}$ L'énoncé est curieux si l'on garde son sens originel, à savoir celui d'une causale enchâssée.

${ }^{6}$ Cette propriété opposant parce que à puisque apparaît également dans R. Martin (1973), J.-C. Anscombre (1984) et

A. Nazarenko (2000).
} 
En revanche, avec parce que, deux interprétations sont possibles pour l'énoncé :

4- J'ai peur qu'il ne l'épouse parce qu'elle est riche.

Ou bien il y a rupture du bloc, comme pour car et puisque, ce qui correspond à l'interprétation : « j'ai peur qu'il ne l'épouse et la raison de ma crainte est l'argent de sa fiancée ». Ou bien il y a conservation du bloc et l'interprétation est alors : « j'ai peur que la cause de son mariage ne soit l'argent de sa fiancée »?

\section{2- Confrontation avec l'analyse d'un corpus attesté}

Bien que cette distinction entre parce que d'un côté et prisque et car de l'autre semble parfaitement établie, il s'avère que l'analyse d'un corpus attesté et non plus forgé change quelque peu les données. En effet, on a pu constater l'existence d'énoncés dont la proposition causale, introduite par puisque, vu que, étant donné que, mais également car, entre bien dans la portée d'un enchâssement. Le bloc « A conj. B » peut ainsi être inséré dans une complétive :

5- Il me semblait que, puisque eux dormaient, Tsurukawa devait dormir. (P. Roze, Le chasseur zéro, p.93)

6- Je lui ai dit que puisque c'était comme ça j'irai sans elle. (F. Seguin, L'arme à gauche, p.88) 7-0n pourrait répondre superficiellement que, étant donné que les colorados ne disposent pas d'une majorité parlementaire, ils sont bien obligés de lancer des ponts vers les autres. (Le Monde diplomatique, mars 1985)

8- Faut dire qu'elle mérite bien qu'j'y consacre une chanson, vu que j'suis amoureux d'elle. (Renaud, Mistral gagnant)

9- Je compris qu'il faudrait cacher ce deuxième regard sur les choses car il ne pourrait susciter que les moqueries de la part des autres. (A. Makine, Le testament français, p.59)

Les trois premiers exemples sont de la même forme que l'exemple (2d) : la causale est antéposée à l'intérieur de la complétive. De plus, on peut supprimer l'enchâssante C, sans modifier l'interprétation sémantique qui s'établit entre A et B. Tel est également le cas en (8) et (9), et bien qu'une pronominalisation de A seul semble grammaticalement possible :

8b- Faut le dire vu que j'suis amoureux d'elle.

9b- Je le compris car il ne pourrait susciter que les moqueries de la part des autres.

Il apparaît que dans ce cas, la causale est clairement sentie comme l'explication de Faut dire que $B$ et Je compris que B, alors qu'en (8) et (9), une ambiguité est ajoutée: la causale peut s'appliquer seulement à A, c'est-à-dire le fait qu'elle mérite une chanson et la nécessité de cacher ce deuxième regard.

Une proposition causale peut également apparaître à l'intérieur d'une proposition circonstancielle :

10- L'embêtant : c'est pas simple à pratiquer parce que si tu y vas mollo tu rates ton coup vu que personne devine ce que tu as derrière le crâne et si tu y vas trop fort c'est plus de l'allusion. (F. Seguin, L'arme à gauche, p.27)

${ }^{7}$ Ibid. p.253. 
Sophie Hamon

11- Comme il savait où elle était, car il ne doutait pas qu'elle fût allée vadrouiller, et pis, avec Paradis, une jubilation se mit à bouillonner en lui, qu'il eut peine à contenir. (R. Queneau, Pierrot mon ami, p.119)

Il n'est pas possible d'appliquer ici les tests de pronominalisation ou de suppression, puisqu'il s'agit d'une circonstancielle et que, en conséquence, elle est dépendante de C. Mais, pour l'exemple (10), on remarque que la causale ne peut pas être antéposée à l'ensemble « $\mathrm{C}+\mathrm{B}$ » :

10b- *Vu que personne devine ce que tu as derrière le crâne c'est pas simple à pratiquer parce que si tu y vas mollo tu rates ton coup et si tu y vas trop fort c'est plus de l'allusion. ${ }^{8}$

De même, pour l'exemple (11), chacun sait qu'une proposition introduite par car ne peut en aucune façon être antéposée, elle ne peut donc, dans cet exemple, que s'appliquer à la circonstancielle en comme.

Enfin, on constate le même phénomène dans une relative :

12- Après la séance triomphale, Jacques plaqua ses copains et se glissa vers les artistes qui l'accueillirent en frère car ils subodoraient une tournée, qu'il offrit. (R. Queneau, Loin de Rueil, p.133)

13- On verra ça, reprit Petit-Pouce qui ne perdait pas sa piste, car il aimait la compétition. (R. Queneau, Pierrot mon ami, p.10)

14- On dit de lui que les ans et la vie retirée l'avaient rendu aussi aveugle que son maitre, fait que je n'ai pas pu vérifier, vu que, par déférence et discrétion naturelle, je ne me suis pas approché suffisamment de sa personne. (Le Monde diplomatique, décembre 1986)

L'enchâssement est donc possible pour toutes les conjonctions causales, bien qu'il ne soit pas systématique. L'opposition entre phrases forgées et phrases attestées illustre cet aspect non systématique et prouve ainsi, d'une part, qu'il y a bien une différence de comportement entre parce que et les autres conjonctions mais, d'autre part, que celle-ci est, en fait, plus complexe et plus subtile qu'une simple propriété formelle.

La confrontation entre les énoncés (4) et (5) à (14) laisse penser que cette différence se situe au niveau de l'interprétation sémantique des enchâssantes et des enchâssées. Autrement dit, pour résoudre ce problème, il s'avère nécessaire de procéder à une analyse sémantique des valeurs causales susceptibles d'être exprimées en langue par ces conjonctions 9 .

\section{Les différentes valeurs causales}

A partir des travaux linguistiques traitant des principales conjonctions causales ${ }^{10}$, nous avons pu formuler une hypothèse globale sur les différentes valeurs introduites par ces conjonctions. Celle-ci repose essentiellement sur une opposition entre contenu, énoncé et énonciation ${ }^{11}$. L'analyse d'un

\footnotetext{
${ }^{8}$ En revanche, on peut tout à fait dire : c'est pas simple à pratiquer parce que vu que personne devine ce que tu as derrière le crâne, si tu y vas mollo tu rates ton coup et si tu y vas trop fort c'est plus de l'allusion, c'est-à-dire antéposer la causale à $B$.

${ }^{9}$ En ce qui concerne les valeurs de ces conjonctions en discours, cf. Moeschler (1996).

10 Il s'agit pour l'essentiel des travaux de R. Martin (1973), J. Hanse (1973), Groupe $\lambda$-1 (1975), J.-C. Anscombre (1984),

L. Danlos (1988), D. Leeman (1994) et A. Nazarenko (2000).

11 Cf. E. Sweetser (1984) qui différencie le domaine du contenu, le domaine épistémique et celui des actes de langage.
} 
corpus d'énoncés attestés (comprenant une cinquantaine d'occurrences pour chaque conjonction et tiré pour l'essentiel du corpus Frantex du CNRS ${ }^{12}$ ) a donc pris appui sur cette hypothèse globale, en se posant pour chaque énoncé les questions suivantes :

1- Est-ce que le fait B produit effectivement le fait A ?

2- Est-ce que B est la cause effective du fait A, de l'énoncé A ou de l'énonciation (i.e. de l'acte de langage contenu en) A?

3-Y a-t-il prise en compte de l'auditeur ou bien intervention de l'énonciateur?

De cette analyse découlent trois principales valeurs causales ${ }^{13}$.

La valeur explicative est de l'ordre du contenu. Les contenus de A et de B présentent deux faits réels et objectifs. La relation qui s'établit entre A et B est telle que B produit effectivement A. Cette production est créé par la « force des choses », elle découle d'une loi naturelle, indépendamment du locuteur ou du sujet de l'énoncé. Seule la conjonction parce que illustre cette valeur :

15- Je ne connaissais que Chopin parce que Nathalie adorait Chopin.

16- Un jour, elle m'enferma dans le cagibi parce que j'avais cassé un vase.

Les autres conjonctions causales peuvent syntaxiquement se substituer à parce que dans ces exemples (Je ne connaissais que Cho pin, puisque Nathalie adorait Chopin; Elle m'enferma dans le cagibi car javais cassé un vase...) mais la valeur sémantique est, dans ce cas, modifiée : l'idée d'un raisonnement intervient automatiquement, la valeur causale exprimée est alors démonstrative.

La valeur démonstrative est à cheval entre le domaine du contenu et celui de l'énoncé, ce dernier étant considéré comme le domaine purement épistémique. A est avant tout une constatation, qui est l'aboutissement d'un raisonnement de la part du locuteur, justifié par B. Autrement dit, si A et $B$ peuvent être considérés simplement en tant que fait, la relation causale qui les unit est indirecte ${ }^{14}$ et découle d'un raisonnement de la part du locuteur. Celle-ci est illustrée par toutes les conjonctions causales :

17- Les branches sont devenues trop diverses parce que les métiers ont évolué.

18- Je dormais peu car avec les pilules roses j'avais jeté mes somnifères.

19- Bruno ne les verrait pas puisque mes cheveux les dissimulaient.

20- On m'a pris à cause de mon bras vu que je ne sais pas faire autre chose qu'écrire.

21- Impossible d'évaluer le nombre de victimes déjà subies par ces villes, étant donné que ni les Américains ni M. Saddam Hussein n'ont quelque intérêt à les divulguer.

La valeur argumentative appartient au domaine de l'énoncé, au domaine épistémique. A n'est pas un fait réel, produit par B, ni une constatation légitimée par B, mais une réflexion du locuteur, une pensée exprimée, justifiée par le fait $\mathrm{B}$; parce que serait curieux dans tous ces exemples, et cela est sans doute dû au fait que la cause et la conséquence semblent avoir été interverties ${ }^{15}$ :

\footnotetext{
12 Les références de ces phrases et les citations complètes se trouvent dans l'Annexe de S. Hamon (2000).

13 La valeur appartenant au domaine de l'énonciation, qui est telle que B est une justification d'un acte d'énonciation (ex. Quiest-ce que tu fais ce soir, parce quill y a un bon film au cinéma? ; Débarrasse, puisque tu n'as rien d'autre à faire.), a été écartée de cet exposé de par son caractère particulier. Pour une analyse plus précise de cette valeur, cf. S. Hamon (2000).

14 En ce qui concerne la relation causale directe et indirecte, cf. L. Danlos (1988).

15 Il s'agit ici du cas où l'acceptabilité de parce que reste discutée dans des phrases comme : ?ll est content parce qu'il sourit, ce qui n'est pas le cas avec puisque ou car : Il est content car / puisquill sourit. Avec parce que, la valeur
} 
Sophie Hamon

23- Peut-être mit-elle sa menace à exécution car pendant près de dix ans maman ne sortit plus. 24- Une journée pas commencée depuis longtemps puisque les magasins étaient tous en train de s'ouvrir.

25- C'était pas non plus la police, vu que le soleil n'était pas encore levé.

26- Une solution qui s'appuierait sur le système français me parait plutôt improbable, étant donné qu'il s'agit d'un système purement national.

La différence entre la valeur démonstrative et la valeur argumentative repose sur l'endroit où intervient le raisonnement : dans le premier cas, il y a uniquement raisonnement au niveau de la relation causale, dans le deuxième cas, le raisonnement apparâtt déjà sous $\mathrm{A}$.

\section{4- La portée de l'enchâssement selon les différentes valeurs causales}

Si une phrase est constituée d'une proposition A et d'une causale B, la valeur causale exprimée par l'énoncé dépend de l'interprétation sémantique de $\mathrm{A}$ et de $\mathrm{B}$. On considère que $\mathrm{B}$ exprime toujours un fait objectif ou du moins que la présence d'une conjonction causale conduit à l'interpréter comme telle. Ainsi, en (35), l'expression je pense est interprétée comme une réflexion du locuteur, alors qu'en (36) cette même expression, enchâssée, semble plutôt traduire l'expression d'un fait :

35- Je pense que l'homme est bon.

36- Je suis naïve parce que je pense que l'homme est bon.

En revanche, comme on l'a vu, la proposition A peut prendre différentes formes interprétatives, elle peut être :

A1 : un fait objectif, la relation causale est alors interprétée comme s'inscrivant dans le monde réel, objectif.

A2 : un fait constaté par le locuteur, c'est-à-dire se situant à la frontière entre le fait et la réflexion. Dans ce cas, la relation causale découle d'un raisonnement qui s'appuie sur deux faits.

A3 : une réflexion du locuteur, explicite ou implicite, du moins posée comme telle.

Une fois ces valeurs déterminées, il a été constaté une répartition relativement stable entre la valeur causale attribuée à un énoncé et la possibilité ou non pour la causale d'être enchâssée. Cette analyse est exposée, par un souci de clarté, à partir de trois phrases construites, représentant les trois valeurs initiales :

- la valeur explicative, représentée par un énoncé de type $\mathrm{e}^{1}(=\mathrm{A} 1+\mathrm{B})$ :

$\mathrm{e}_{1}$ - Julie est malade parce qu'elle a attrapé froid.

- la valeur démonstrative, exprimée par un énoncé de type $\mathrm{e}^{2}(=\mathrm{A} 2+\mathrm{B})$ :

$\mathrm{e}_{2}$ - Julie sera malade parce que / car / puisqu'elle a attrapé froid. ${ }^{16}$

- la valeur argumentative, exprimée par un énoncé de type $\mathrm{e}^{3}(=\mathrm{A} 3+\mathrm{B})$ :

causale est transposée : A n'est plus considéré en tant que contenu, ni en tant qu'énoncé, mais en tant qu'énonciation ; ce n'est pas le raisonnement A qui est justifié mais le fait de dire A et par extrapolation de le penser, c'est pourquoi cet énoncé peut être accepté.

${ }^{16}$ Le simple emploi du futur permet de caractériser l'événement A comme un fait imaginé et donc de le situer véritablement à la frontière entre fait et réflexion. 
$e_{3^{-}}$Julie a peut-être attrapé froid hier soir puisque / car elle est malade.

Chacun de ces trois énoncés subit différemment la portée de l'enchâssement selon l'information sémantique véhiculée par l'enchâssante C. Nous proposons d'analyser ici les trois interprétations principales que cette dernière est susceptible d'exprimer, à savoir :

C1: un fait réel, objectif ; une telle proposition enchâsse généralement une relative ou une proposition circonstancielle: Lucien appelle Julie, qui A conj B; Comme A conj B, Lucien appelle Julie.

C2: un acte de discours; il s'agit alors du cas d'un enchâssement dans un discours indirect: Lucien dit que A conj $B$.

C3: une réflexion explicite du locuteur; elle permet de caractériser les enchâssements dans une complétive de type Lucien pense que A conj B.

L'analyse consiste à examiner les différentes combinaisons possibles $\left(\mathrm{C} 1+\mathrm{e}^{1} / \mathrm{e}^{2} / \mathrm{e}^{3} ; \mathrm{C} 2+\right.$ $\left.\mathrm{e}^{1} / \mathrm{e}^{2} / \mathrm{e}^{3} ; \mathrm{C} 3+\mathrm{e}^{1} / \mathrm{e}^{2} / \mathrm{e}^{3}\right)$, en se demandant si la causale est ou non comprise dans l'enchâssement.

\section{4-1-Enchâssement par une proposition Cl (fait objectif)}

Si la proposition enchâssante $C$ exprime un fait réel, ou du moins si elle est utilisée à cette seule fin, alors, quelle que soit la valeur causale exprimée par A et B et quelle que soit la conjonction utilisée, la causale est toujours interprétée comme étant incluse dans l'enchâssement :

37- Lucien a appelé Julie, qui est malade parce qu'elle a attrapé froid hier soir. (= C1, A1 conj B) 38- Lucien a appelé Julie, qui sera malade parce que / car / puisqu'elle a attrapé froid hier. (= C1, A2 conj B)

13- On verra ça, reprit Petit-Pouce qui ne perdait pas sa piste, car il aimait la compétition. (= C1, A2 conj B)

39- Lucien a appelé Julie, qui a peut-être attrapé froid hier soir, puisque / car elle est malade. ( = C1, $\mathrm{A} 3 \operatorname{conj} \mathrm{B})$

40- Comme Julie est malade, parce qu'elle a attrapé froid hier soir, je vais appeler le médecin. (= Comme A1, conj B, C1)

41- Comme Julie sera malade, parce que / car / puisqu'elle a attrapé froid hier soir, je vais appeler le médecin. (= Comme A2, conj B, C1)

10- L'embêtant : c'est pas simple à pratiquer parce que si tu y vas mollo tu rates ton coup vu que personne devine ce que tu as derrière le crâne et si tu y vas trop fort c'est plus de l'allusion. (= $\mathrm{C} 1$ parce que $\mathrm{A} 2$ conj $\mathrm{B})$

42- Comme Julie a peut-être attrapé froid hier soir, puisque / car elle est malade, je vais monter le chauffage. (= Comme A3, conj B, C1)

Les exemples (11) et (12) du corpus attesté illustrent également cette propriété.

On peut néanmoins noter que cette propriété est valide à condition que la relation entre A et B soit plus « logique » ${ }^{17}$ au regard de l'interprétant que ne l'est celle entre $\mathrm{C}$ et B. Ainsi, à ce titre, la comparaison entre (43) et (44) montre qu'il est nécessaire de n'avoir aucun lien logique entre B et A, mais bel et bien entre $\mathrm{C}$ et $\mathrm{A}$, pour que la causale ne soit pas considérée comme enchâssée :

43- Lucien a appelé Julie, qui est malade, parce qu'il voulait l'inviter au cinéma.

${ }^{17}$ Le terme logique exprime, dans ce cas, uniquement l'idée intuitive d'une interprétation plus naturelle. 
Sophie Hamon

44- Lucien a appelé Julie, qui doit sûrement être malade, puisqu'elle n'était pas au rendez-vous.

En (43), la causale ne peut s'appliquer logiquement qu'à la proposition C, donc elle est bien interprétée comme non-enchâssée. En (44), la proposition B peut s'appliquer aussi bien à C qu'à A, pourtant l'interprétation la plus naturelle sera de la considérer comme enchâssée et donc ne portant que sur $A$.

\section{4-2- Enchâssement par une proposition C2 (acte de discours)}

En ce qui concerne le cas où un énoncé $e$ est enchâssé dans un discours indirect, on constate que la portée de l'enchâssement varie en fonction de la valeur causale exprimée en $e$. Lorsque la valeur causale est explicative, que seul parce que exprime, alors B est plus naturellement considéré comme enchâssé, comme le montrent les énoncés (45) et (15b) :

45- Je lui ai dit que Julie est malade parce qu'elle a attrapé froid hier soir. (= C2 que A1 conj B)

15b- Je lui ai dit que je ne connaissais que Chopin parce que Nathalie adorait Chopin.

Seule une intonation marquée permet de détacher B de l'ensemble $(C+A)$.

Parce que est la seule conjonction qui puisse établir une relation causale objective entre deux faits. Dans les exemples (45) et (15b), C n'étant pas présenté comme un fait mais comme un acte de discours, le locuteur interprète plus naturellement B comme la cause du fait A. Avec une intonation particulière qui signale que $C$ et $A$ sont liés, alors l'ensemble ainsi formé est considéré comme un fait - celui de dire B - et donc B peut en être la cause. Encore une fois, la proposition B est considérée comme non enchâssée dans les cas où elle a un lien logique plus fort avec $\mathrm{C}$ qu'avec $\mathrm{A}$ :

46- Je lui ai dit que Julie est malade parce qu'elle me l'a demandé.

Pour les valeurs démonstrative et argumentative, il est difficile de choisir entre l'une ou l'autre option. Il semble que les deux soient possibles, dans le sens où le locuteur peut tout à fait avoir prononcé l'énoncé $e$ en entier, le discours indirect gardant alors exactement la même forme que le discours direct.

47- Je lui ai dit que Julie sera malade parce que / car / puisqu'elle a attrapé froid hier soir. (= C2 que A2 conj B)

48- Je lui ai dit que Julie a peut-être attrapé froid hier soir puisque / car elle est malade. (= C2 que A3 conj B)

18b- Je lui ai dit que je dormais peu car avec les pilules j’avais jeté les somnifères. (=C2 que A2 conj B)

19b- Je lui ai dit que Bruno ne les verrait pas puisque mes cheveux les dissimulaient. (=C2 que A2 conj B)

25b- Je lui ai dit que c'était pas non plus la police, vu que le soleil n'étais pas encore levé. (=C2 que A3 conj B)

Sachant que A2 peut aussi bien être compris en tant que fait qu'en tant que réflexion, l'introduction d'un discours indirect (C2) semble privilégier la deuxième considération. De ce fait, dans le cas de la valeur démonstrative, parce que conduit à interpréter B comme la cause d'un tel acte de discours, soit de l'ensemble $\mathrm{C}+\mathrm{A}$ (ex.47), alors que les autres conjonctions amènent davantage à interpréter $\mathrm{B}$ comme la raison qui justifie, en amont, le raisonnement du locuteur.

Pour faire comprendre que B est bien enchâssé et donc qu'il apparaissait dans le discours direct, le locuteur a la possibilité de placer B avant A, ce qui lève l'ambiguiité : 
47b- Je lui ai dit que parce que / puisqu'elle a attrapé froid hier soir, Julie sera malade.

\section{4-3- Enchâssement par une proposition C3 (réflexion)}

Lorsqu'on enchâsse une proposition $e$ dans une proposition exprimant explicitement une réflexion du locuteur (C3), alors, dans ce cas, seul parce que autorise d'être dans la portée de $\mathrm{C}$. Une expression du type Je pense que A peut exprimer le fait réel de penser A ou bien la réflexion A que le locuteur s'attribue. C'est la différence d'interprétation du verbe penser que l'on trouve en comparant les énoncés (49) et (50) à l'énoncé (51) :

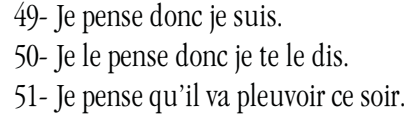

parce que a comme valeur sémantique de faire interpréter la proposition, dont il introduit la cause, comme exprimant un fait réel. De ce fait, deux cas de figure sont possibles avec cette conjonction : ou bien la proposition B est la cause du fait de penser A, elle n'est donc pas enchâssée ; ou bien B est simplement la cause du fait A, elle est donc enchâssée.

52- Je pense que Julie est malade parce qu'elle a attrapé froid hier soir. (C3 que A1 conj B)

La différence d'interprétation entre les deux est très étroite et donc va dépendre de l'interlocuteur. Si celui-ci connaît le fait que Julie est malade, alors la causale sera automatiquement comprise comme ne portant que sur A. En effet, le locuteur n'a pas à justifier le fait de penser A, si A est déjà admis de l'interlocuteur. En revanche, il peut chercher à donner une explication possible du fait A lui-même. Si l'interlocuteur ignore tout de la maladie de Julie, B va être plus naturellement ressenti comme la cause de l'ensemble $\mathrm{C}+\mathrm{A}$, à savoir le fait que le locuteur pense $\mathrm{A}$ :

53- - Il faut absolument que j'appelle Julie, ça fait longtemps que je ne l'ai pas vue.

- Oui, mais tu sais, je pense que Julie est malade aujourd'hui, parce qu'elle a attrapé froid hier soir.

Cela explique ainsi que dans le cas où la valeur démonstrative est exprimée, la causale, même avec parce que, portera naturellement sur l'ensemble $\mathrm{C}+\mathrm{A}$ : comme $\mathrm{A}$ est une réflexion ou un fait futur, il ne peut pas déjà être connu et donc $B$ sera la cause du fait de penser $\mathrm{A}^{18}$ :

54- Je pense que Julie sera malade parce qu'elle a attrapé froid hier soir. (= C3 que A2 conj B)

Les autres conjonctions causales contiennent toutes en elles-mêmes l'idée d'un raisonnement de la part du locuteur - c'est pourquoi aucune d'entre elles ne figure dans ce que nous appelons la valeur explicative. Lorsqu'elles introduisent une valeur démonstrative, pour laquelle il y a ambiguïté entre fait et réflexion, il y a également ambiguiité entre enchâssement et détachement.

55- Je pense que Julie sera malade puisqu'elle a attrapé froid hier soir. (= C3 que A2 conj B) 5- Il me semblait que, puisque eux dormaient, Tsurukawa devait dormir. (= C3 que A2 conj B)

Ainsi, dans l'exemple (5), il y a une double interprétation:

\footnotetext{
18 Dans le cas d'une reprise de A, alors la causale peut ne porter que sur le fait A : Je pense que Julie sera malade parce quielle a attrapé froid hier soir et non parce quelle a mangé tout le chocolat, comme tu le laisses entendre.
} 
a) Tsurukawa dort parce que les autres dorment.

b) Comme les autres dorment, j'estime très probable que Tsurukawa dorme aussi.

L'interprétation (a) est liée à la possibilité de considérer la causale comme étant enchâssée, elle disparaît d'ailleurs en plaçant la causale hors de l'enchâssement :

5b- Puisque eux dormaient, il me semblait que Tsurukawa devait dormir.

L'interprétation (b), liée au détachement de la causale, est présente partiellement en (5), bien que la causale soit à l'intérieur de la complétive, parce que la principale Il me semblait que ne fait qu'expliciter ce que la modalité présente en A (Tsurukawa devait dormir) présupposait, à savoir que A est une réflexion du locuteur légitimée par le fait $\mathrm{B}^{19}$. C'est pourquoi l'ambiguité ne gêne pas la compréhension de la phrase.

Cela permet également de comprendre pourquoi dans le cas de l'expression d'une valeur argumentative, la causale est obligatoirement comprise comme étant détachée.

56- Paul croit que Julie a peut-être attrapé froid hier soir car / puisqu'elle est malade. (=C3 que A3 conj B)

25b- Paul comprit que ce n'était pas la police vu que le soleil n'était pas encore levé. (= C3 que A3 conj B)

La proposition du type C3 ne fait que souligner explicitement le fait que A est une réflexion du locuteur. Si la causale s'applique à $\mathrm{A}$, alors elle s'applique tout autant à $\mathrm{C}$, qui ne fait que décrire explicitement la forme de la réflexion A (doute, croyance, certitude...). Donc une causale introduite par puisque, car ou vu que ne peut que porter sur l'ensemble $(\mathrm{C}+\mathrm{A})$, dans un tel enchaînement, soit être hors de l'enchâssement.

\section{5- Conclusion}

L'analyse a montré que lorsque l'enchâssante décrit un fait réel (C1), l'enchâssement est possible pour toutes les conjonctions, à condition que la relation unissant B à A soit plus «logique » que celle unissant B à C. Si C représente un acte de discours (C2), alors, il y a les deux possibilités pour toutes les conjonctions ; ce sont le contexte et l'intonation donnée à l'énoncé qui permettent de savoir où s'arrête le discours rapporté. Enfin quand l'enchâssante est de type C3, c'est-à-dire exprimant une réflexion du locuteur, alors, dans ce cas seulement, parce que est la seule conjonction susceptible d'être enchâssée. Mais cela s'explique par la valeur sémantique de la conjonction par rapport à celle des autres : s'il y a expression d'un fait réel en A, alors il y a enchâssement, s'il y a expression d'une réflexion en $\mathrm{A}$, alors il y a détachement, puisqu'on ne peut pas dans ce cas dissocier A de C. Parce que est la seule conjonction qui conduise à interpréter véritablement A comme un fait, c'est donc la seule conjonction qui puisse être enchâssée dans une complétive de type C3.

\footnotetext{
${ }^{19}$ La même analyse peut être faite pour l'exemple (9), l'ambiguïté entre enchâssement ou détachement de la causale découle de la double interprétation de $\mathrm{A}$ : le fait de cacher ce deuxième regard sur les choses et la nécessité de le faire, cette nécessité étant une réflexion du locuteur, qui est explicitée par la proposition C « je compris que ». En (55), la causale explique 1) le fait que Julie puisse tomber malade, et 2) la supposition du locuteur - quant à la réalisation de cet événement - exprimée par l'emploi du futur, et explicitée par la principale Je pense que.
} 
Les exemples sur lesquels s'appuie le groupe $\lambda$-1 (exemples (3a) et (3b)), pour démontrer le non-enchâssement de puisque ou de car, illustrent justement ce troisième cas : $\mathrm{C}$ explicite que $\mathrm{B}$ est bien un raisonnement ; en toute logique, la causale portera donc sur l'ensemble $C+A$, puisqu'en quelque sorte $\mathrm{C} 3$ est déjà compris en $\mathrm{A} 3$. Leur analyse est donc valide dans ce cas (la causale ne peut pas être interprétée comme enchâssée dans un énoncé du type « $\mathrm{C} 3$ que $\mathrm{A} 3$ conj $\mathrm{B}$ »), mais elle ne peut pas être utilisée pour établir une généralité.

La propriété de l'enchâssement ne peut pas être considérée comme une simple propriété formelle qui distingue parce que de car ou de puisque. Toutes ces conjonctions sont susceptibles d'entrer dans la portée d'un enchâssement, ce sont les valeurs sémantiques rattachées à l'énoncé qui autorisent ou non l'enchâssement.

Sophie HAMON

Université de Paris X- Nanterre

UMR MODYCo 7114

\section{RÉFÉRENCES}

ANSCOMBRE, J-C. (1984), « La représentation de la notion de cause dans la langue », Cabiers de grammaire, $\mathrm{n}^{\circ} 8$, Université de Toulouse - Le Mirail.

DANLOS , L. (1988), « Connecteurs et relations causales », Langue française, $n^{\circ} 77$, Paris, Larousse.

GOSSELIN , L. (1993) , «Circonstanciels et relatives : stratégie d'analyse syntaxique », in Cl. Guimier (éd.) 1001 Circonstants, Presses universitaires de Caen.

GROUPE $\lambda-1$ (1975), «Car, parce que, puisque», Revue romane, t. X, n², Copenhague Munksgaard. International Publishus.

GREVISSE, M. (1986), Le bon usage, Paris-Gembloux, Duculot.

HAMON, S. (2000), L'expression de la cause à travers les phénomènes de coordination et de subordination: analyse des principales conjonctions causales, mémoire de DEA, Université Paris X-Nanterre.

HANSE, J. (1973), «Car, comme, parce que, puisque», Bulletin de l'Académie royale de langue et de littérature française, $\mathrm{n}^{\circ} \mathrm{LI}: 3-4$, Belgique.

LEEMAN , D. (1994), « Remarques sur puisque et sur car »,Théories, données et pratiques en français langue étrangère, Presses universitaires de Lille.

MARTIN, R. (1973), «Le mot puisque : notion d'adverbe de phrase et de présupposition sémantique », Studia Neophilologica, vol. XLV, $n^{\circ} 1$, Stockholm, The Almqvist \& Wiksell Periodical Company.

MOESCHLER, J. (1996),Théorie pragmatique et pragmatique conversationnelle, Paris, Colin.

NAZARENKO, A. (2000), La cause et son expression en français, Paris, Ophrys.

RIEGEL, M. et al. (1994), Grammaire méthodique du français, Paris, PUF.

SWEETSER, E. (1984), Semantic structure and semantic change : a cognitive linguistic study of modality, perception, speech acts, and logical relations, Ph. D. Dissertation, University of California, Berkeley. 
